\title{
Kinetics of 'cluster eating'
}

\author{
S Redner, D Ben-Avraham and B Kahng \\ Center for Polymer Studies and Department of Physics, Boston University, Boston, MA \\ 02215 , USA
}

Received 8 May 1986

\begin{abstract}
We introduce and study the kinetics of 'cluster eating', in which a cluster of mass $i$ and a cluster of mass $j$ react to form a lighter cluster of mass $|i-j|$. We write the rate equations for this process, which describe the kinetics in the mean-field limit, where spatial fluctuations in cluster density and in cluster shape are neglected. An asymptotic solution to these equations is derived for the particular case in which the reaction rate is independent of the masses of the reacting clusters. At long times, we find that the density of clusters of mass $k, c_{k}(t)$ decays as $A_{k} /\left(\tau \log ^{k-1} \tau\right)$, where $\tau$ is proportional to the time, $A_{k}=(N-1) ! /(N-k) !$ and $N$ is the largest cluster mass in the initial state. This very unusual behaviour is checked by numerical simulations. A more general situation where the reaction matrix depends on the parities of the masses of the two incident clusters is also discussed briefly and a wide variety of possible kinetic behaviours is delineated. Finally, we study cluster eating below the upper critical dimension, where fluctuations in cluster density give rise to a non-classical kinetic behaviour.
\end{abstract}

The investigation of the kinetics of reacting systems is an area of study that has recently been the focus of considerable attention (see, e.g., Noyes 1961, Calef and Deutch 1983, Kang and Redner 1985a, Zumofen et al 1985 and references therein). In addition to the obvious practical and technological applications of a variety of reaction schemes, many such processes are also of considerable intrinsic theoretical interest from the viewpoint of understanding non-equilibium phenomena at a fundamental level. One very important example of a reacting system is the phenomenon of coagulation, in which clusters irreversibly bond, upon colliding, to form clusters of perpetually increasing size (Ernst 1985, van Dongen and Ernst 1985, Leyvraz 1986). This process can be described by the mass conserving scheme, $c_{i}+c_{j} \rightarrow c_{i+j}$, where $c_{k}$ denotes a cluster of mass $k$. From a biological description, coagulation can be regarded as a cooperative pairwise merging of two separate population centres to form a single larger centre. Coagulation has been found to exhibit a wealth of kinetic behaviour, both at the level of a rate equation approximation, and also when spatial fluctuations in cluster density are taken into account (Kang and Redner 1985b).

In this paper, we introduce a very simple kinetic process, which we term 'cluster eating', in which two population centres react in a destructively interfering manner. This model was inspired, in part, as an attempt to formulate models of competing biological species which vere as simple as possible. While our model is perhaps too crude to have immediate practical applications, the kinetics of the model is quite intriguing, and therefore appears to possess intrinsic theoretical interest. On a very superficial level the mathematical description of cluster eating has some resemblance with that of coagulation, but there are important differences between the two models which are the source of a variety of novel kinetic phenomena. 
Our model is defined by the reaction scheme, $c_{1}+c_{j} \rightarrow c_{k}$, with $k=|i-j|$ as illustrated in figure 1 . When two clusters meet, individual members of the two clusters can be regarded as annihilating in pairs, leaving behind a residue consisting of the difference of the masses of the two incident clusters. We are interested in the kinetics of this process, primarily in the mean-field, or rate equation, approximation. For the generic case where the reaction rates are independent of the masses of the reacting clusters (constant matrix of reaction rates) we show that the density of clusters of mass $k$ at time $t, c_{k}(t)$, varies at long times as $A_{k} /\left(\tau \log ^{k-1} \tau\right)$, where $\tau$ is proportional to the time, and the amplitude $A_{k}$ is equal to $(N-1) ! /(N-k) !$, with $N$ being the mass of the largest cluster present initially. This peculiar behaviour, replete with logarithmic corrections, is quite unlike that found for the kinetics of the more familiar bimolecular reaction processes such as coagulation and recombination. We have verified this novel behaviour by numerical simulations.

We also consider a more general version of cluster eating in which the reaction rate depends on the relative parity of the masses of the two incident clusters. A wide variety of kinetic behaviours can occur and we briefly outline the range of possibilities. We also investigate the kinetics of cluster eating in a system of spatial dimension $d$ less than the upper critical dimension $d_{c}$, where fluctuations in cluster density will influence the reaction kinetics. In one dimension, the total cluster density is found to decay as $t^{-1 / 2}$, as expected on the basis of appealing to analogies with closely related reactions, $A+A \rightarrow A$ and $A+A \rightarrow$ inert (see, e.g., Torney and McConnell 1983a, b, Toussaint and Wilczek 1983). The temporal behaviour of each of the $c_{k}(t)$ is also discussed.

The mean-field rate equations for cluster eating are

$$
\dot{c}_{k}(t)=\sum_{i=1}^{N-k} K(i, i+k) c_{i}(t) c_{i+k}(t)-c_{k}(t) \sum_{i=1}^{N} K(i, k) c_{i}(t)
$$

where the dot denotes time differentiation. The first sumriation accounts for the production of clusters of mass $k$ due to the reaction of two clusters whose mass difference is equal to $k$, while the second summation accounts for the loss of $k$ clusters due to their reaction with other clusters in the system. The matrix of rate constants, $K(i, j)$, describes the dependence of the reaction rate on the masses $i$ and $j$ of the two incident clusters. We shall primarily be interested in the relatively simple case of a constant reaction matrix.

There are several features of the rate equations that are worth noting. First, unlike the case of coagulation, the upper limits in both sums are finite, corresponding to a finite value, $N$, of the maximum cluster mass in the initial population distribution.

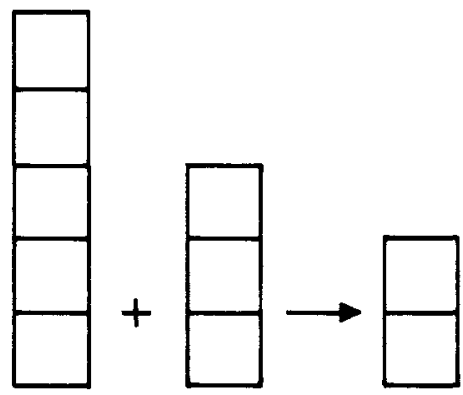

Figure 1. Typical reaction in the cluster eating model. 
Also notice that the cluster eating process does not conserve mass and the production term is not of the form of a convolution. As a consequence, standard generating function or Laplace transform methods, which are of great utility in mass-conserving processes (see, e.g., Drake 1972), have limited utility in cluster eating.

Before presenting our asymptotic solution to the rate equations for cluster eating, we discuss a number of interesting general aspects of the problem. For the case of a constant reaction matrix, one can easily show from the rate equations that the total density of clusters, $\rho(t) \equiv \Sigma_{k} c_{k}(t)$, obeys the differential equation

$$
\sum_{k=1}^{N} \dot{c}_{k}(t)=-\frac{1}{2}\left(\sum_{k=1}^{N} c_{k}(t)\right)^{2}-\frac{1}{2} \sum_{k=1}^{N} c_{k}(t)^{2}
$$

where the reaction rate has been chosen to be equal to unity. Since $\Sigma c_{k}(t)^{2}$ satisfies the obvious bounds $0 \leqslant \Sigma c_{k}(t)^{2} \leqslant\left(\Sigma c_{k}(t)\right)^{2}$, we can use this in (2) to conclude that, at long times, $\rho(t)$ obeys the bounds, $1 / t<\rho(t) \leqslant 2 / t$. A decay of the total cluster density proportional to $1 / t$ is to be expected since, when two clusters of mass $i$ and $j$ meet, the product may be either a single cluster of mass $|i-j|$, when $i \neq j$, or there may be no product cluster if $i=j$. On this basis, cluster eating can be thought of as a superposition of the single-species reaction schemes, $A+A \rightarrow A$ and $A+A \rightarrow$ inert, and the kinetics of cluster eating should mirror the kinetics of these two reactions.

A second interesting point is that the total density of odd clusters in the system, $\rho_{\text {odd }}(t) \equiv \Sigma_{\text {odd }} c_{k}(t)$, can be obtained exactly. From the rate equations, we find

$$
\dot{\rho}_{\text {odd }}(t)=-\left(\rho_{\text {odd }}(t)\right)^{2}
$$

with solution

$$
\rho_{\text {odd }}(t)=\frac{\rho_{\text {odd }}(0)}{1+\rho_{\text {odd }}(0) t}
$$

This simple result reflects the fact that when two odd clusters meet they both disappear from the system and an even cluster is produced. This is the only mechanism available for changing the density of odd clusters.

Let us now turn to the asymptotic solution of the rate equations. Suppose that the mass of the heaviest cluster in the initial distribution of clusters is $N$. Then the concentration of clusters of mass $N$ satisfies the equation

$$
\dot{c}_{N}(t)=-c_{N}(t) \sum_{i=1}^{N} c_{i}(t)
$$

Thus we can write a formal solution for $c_{N}(t)$

$$
c_{N}(t)=c_{N}(0) \exp \left(-\int_{0}^{t} \sum c_{i}\left(t^{\prime}\right) \mathrm{d} t^{\prime}\right) \equiv c_{N}(0) \mathrm{e}^{-x} .
$$

Since $\Sigma_{i} c_{i}(t)$ is inversely proportional to the time at long times, we conclude that $x$ will vary as $\ln t$ as $t \rightarrow \infty$.

To solve the rate equations for $c_{k}(t)$ when $k<N$, we observe that there is an integrating factor equal to $\exp \left(\int \Sigma c_{i}\left(t^{\prime}\right) \mathrm{d} t^{\prime}\right)$, and from (6), this is simply equal to $c_{N}(0) / c_{N}(t)$. Using this result, the rate equations can now be cast into the form

$$
\frac{\mathrm{d}}{\mathrm{d} t}\left(\frac{c_{k}(t)}{c_{N}(t)}\right)=c_{N}(t)^{-1} \sum_{i=1}^{N-k} c_{i}(t) c_{i+k}(t) .
$$

By introducing the scaled concentration, $\phi_{k}=c_{k}(t) / c_{N}(t)$, and also rescaling the time 
in terms of the auxiliary variable $\mathrm{d} y=c_{N}(t) \mathrm{d} t$, we can rewrite $(7 a)$ as

$$
\phi_{k}^{\prime}=\sum_{i=1}^{N-k} \phi_{i} \phi_{i+k} \quad k=1,2, \ldots, N-1
$$

where the prime denotes differentiation with respect to the variable $y$. In this form, the rate equations possess only production terms and this simplification facilitates an asymptotic solution. The general philosophy underlying this approach to solving the rate equations has already been well established for the coagulation problem (Lushnikov 1973, 1974, 1975, Leyvraz 1984). It is also worth mentioning the possibility of writing the rate equations in terms of a different scaled concentration, namely $\psi_{k}=$ $c_{k} / \rho_{\text {odd }}(t)$, rather than in terms of the $\phi$. This approach has the advantage that the corresponding timelike variable, $\mathrm{d} y=\rho_{\text {odd }}(t) / \mathrm{d} t$, is simply equal to $\ln \left(1+\rho_{\text {odd }}(0) t\right)$ from (4). However, the transformed rate equations are not as simple in form as those of $(7 b)$.

By inspection of $(7 b)$, one finds that the $\phi_{k}$ must exhibit power law singularities at a finite value of $y$ which depends on the initial conditions. If one therefore substitutes the asymptotic form

$$
\phi_{k} \sim \frac{\bar{A}_{k}}{(a-y)^{\alpha_{k}}}
$$

into $(7 b)$, one finds that the most singular contribution to $\phi_{k}^{\prime}$ arises from the term $\phi_{1} \phi_{k+1}$. At this level of approximation, it is then straightforward to show that the exponent $\alpha_{k}$ is

$$
\alpha_{k}=\frac{N-k}{N-2}
$$

and that the corresponding amplitude $\bar{A}_{k}$ is given by

$$
\bar{A}_{k}=\frac{(N-2)^{N-k}}{(N-k) !} \bar{A}_{1}^{N-k} \quad \bar{A}_{1}=\left(\frac{(N-1) !}{(N-2)^{N-1}}\right)^{1 /(N-2)} .
$$

More generally, there will be a series of correction terms in (8) which are all less singular as $y \rightarrow a$.

To find the actual time dependence of the cluster density, we must now write the variable $y$ as a function of time. To accomplish this, note that from the definitions, $\mathrm{d} x=\Sigma c_{i}(t) \mathrm{d} t$ and $\mathrm{d} y=c_{N}(t) \mathrm{d} t$, we have

$$
x=\int \sum_{i=1}^{N-1} \phi_{i} \mathrm{~d} y .
$$

In the same spirit as the approximation that led to (8), we approximate the sum in (10) by its largest term, $\phi_{1}$ to give

$$
x \sim(a-y)^{-1 /(N-2)}
$$

Since we know that $x$ varies as $\ln t$ as $t \rightarrow \infty$, we conclude that $y$ must vary as $a-\varepsilon(t)$ with

$$
\varepsilon(t) \sim(\ln t)^{-(N-2)}
$$

With this information, we can now find the asymptotic behaviour of the cluster density. We have

$$
c_{N}(t)=\dot{y}=-\dot{\varepsilon} \sim \frac{1}{t(\ln t)^{N-1}}
$$


while from $c_{k}=\phi_{k} c_{N}$ we obtain the general result

$$
c_{k}(t) \sim \frac{A_{k}}{t(\ln t)^{k-1}}
$$

Here $A_{k}=(N-1) ! /(N-k)$ !, as can be verified by explicitly keeping track of prefactors in the foregoing analysis and using the expression for the $\bar{A}_{k}$. Strictly speaking, the time $t$ in $(13 b)$ should be the quantity $\tau=1+\rho_{\text {odd }}(0) t$. Thus we conclude that the density of $k$ clusters decays as $1 / t$ but modified by a $k$-dependent logarithmic factor. This very peculiar feature suggests that the model with a constant reaction matrix is in some sense marginal. We shall return to this point below.

One very simple check of this asymptotic solution is to consider the special case $N=2$ for which the exact solution can be found. When $N=2$, the rate equations are

$$
\begin{aligned}
& \dot{c}_{1}(t)=-c_{1}(t)^{2} \\
& \dot{c}_{2}(t)=-c_{1}(t) c_{2}(t)-c_{2}(t)^{2} .
\end{aligned}
$$

By elementary methods, we obtain

$$
\begin{aligned}
& c_{1}(t)=\frac{c_{1}(0)}{1+c_{1}(0) t} \\
& c_{2}(t)=\frac{c_{1}(0)}{1+c_{1}(0) t}\left(\frac{c_{2}(0)}{c_{1}(0)+c_{2}(0) \ln \left(1+c_{1}(0) t\right)}\right)
\end{aligned}
$$

in agreement with the asymptotic form given in (13). While the presence of a logarithmic correction term is perhaps unexpected, a priori, its effect is rather weak. For example, for the $N=2$ case, consider the situation where $c_{1}(0)=c_{2}(0)$. If one measures time in units of $c_{1}(0)^{-1}$, then when $10^{10}$ time units have elapsed, $c_{1}(t) / c_{1}(0)$ is approximately equal to $10^{-10}$, but the ratio $c_{2}(t) / c_{1}(t)$ has decayed to only $\sim \frac{1}{24}$.

The asymptotic solution given in (13) is replete with logarithmic correction factors, in a manner analogous to the logarithmic factors appearing in the description of critical phenomena at the upper critical dimension. A related behaviour in a coagulation model has also been found by Leyvraz (1985). This suggests that cluster eating with a constant matrix of reaction rates is in some sense marginal. Indeed, from the exact solution of the $N=2$ case with an arbitrary matrix of reaction rates, i.e. $K(1,1) \neq K(1,2)$, we see that this is precisely the case. For $K(1,1)>K(1,2), c_{2}(t)$ decays as $1 / t$; for $K(1,1)<K(1,2), c_{2}(t)$ decays as $1 / t^{K(1,2) / K(1,1)}$. The logarithmic corrections appear as the two reaction constants become equal. In general, we expect logarithmic corrections to arise only when there is some special symmetry in the form of the reaction matrix.

On this basis, we are led to consider a more general situation where the reaction matrix is not a constant. The generalisation that we consider is motivated in part by the very rich and intriguing behaviour found in a closely related coagulation model (Leyvraz and Redner 1986, see also Djordjevic and Meakin 1986). We note that the density of odd clusters changes only when two odd clusters meet to form an even cluster. On the other hand, even clusters are produced by the reaction of two odd or two even clusters (with unequal masses), while they are destroyed by both odd-even or even-even (of equal mass) reactions. Therefore it seems natural to classify the various reactions according to the relative parity of the masses of the reacting clusters. 
Thus, suppose that the reaction rate depends on the incident cluster masses as follows:

$$
\begin{aligned}
K(i, j) & =K & & \text { for } i \text { and } j \text { odd } \\
& =L & & \text { for } i \text { and } j \text { even } \\
& =M & & \text { otherwise. }
\end{aligned}
$$

Although such a model should ostensibly be in the same universality class as a model with a constant matrix of reaction rates, the imposed modulation of the matrix evidently breaks, to some extent, the marginal behaviour of the constant matrix model.

Just as in the corresponding coagulation problem, we find a very wide range of possible kinetic behaviours. For $K<M$ we find even weaker logarithmic corrections than in the constant reaction matrix case, namely, both $c_{2 k}$ and $c_{2 k+1}$ decay as $1 / t(\ln t)^{k}$, while when $N$ is even, $c_{N}$ decays as $1 / t^{M / K}$. For $K>M$ we have not yet succeeded in exploring all the possibilities. Depending on initial conditions, as well as on the relative value of $L$, the $c_{k}$ might all decay as $1 / t$, or there might even be cases where at least one of the species decays algebraically slower than $1 / t$. We hope to report more details of the kinetics for this interesting region of the $(K, L, M)$ space. The case of $K=M$ is very much alike that of the constant rate matrix, only that $c_{N} \sim$ $1 / t(\ln t)^{(N-2) K / L}$ when $N$ is odd and $L<[(N-2) /(N-1)] K$ and $c_{N}$ $1 / t(\ln t)^{(N-2) L / K}$ when $N$ is even and $L>[(N-1) /(N-2)] K$.

In order to test the validity of the asymptotic solution, we halve performed numerical simulations of cluster eating in the mean-field limit. The approach employed is quite similar to that used in previous studies of coagulation (Kang et al 1986). A basic feature of this method is that a cluster is defined to occupy a single lattice site and the mass is retained as a scalar variable associated with each occupied site. Initially, clusters are randomly placed on a lattice according to a specified initial mass distribution. To simulate the kinetics of the rate equation, or mean-field approximation, clusters are moved at random to any other site of the lattice with a uniform probability. When two clusters of mass $i$ and $j$ happen to occupy the same lattice site, a new single-site cluster is defined with mass equal to $|i-j|$. If $i=j$, the two clusters annihilate, leaving nothing behind. Note that the lattice structure is irrelevant for this infinite mobility transport mechanism, and therefore we use a linear chain, as it is the most convenient. This general simulation procedure can be straightforwardly extended to study the kinetics of diffusing clusters in low-dimensional systems by replacing the infinite mobility rule with nearest-neighbour hopping.

For most of our simulations, we have considered the specific initial condition of $c_{k}(0)$ all equal to a common constant for $k \leqslant N$ and $c_{k}(0)=0$ for $k>N$. Typical results for the mean-field limit are shown in figure 2. The full lines were plotted using the asymptotic result, equation $(13 b)$. Notice that the densities of the larger clusters converge rather quickly to the behaviour of the asymptotic regime. This phenomenon is explained by the fact that the decay of small clusters is influenced by correction terms which are of the order of the sum of the density of all other larger clusters. Also plotted is the decay of $\rho_{\text {odd }}(t)$, the sum of odd clusters, for which an exact decay law, valid at all times, is known (equation (4)). The agreement between theory and simulations is extremely good.

Now we consider the kinetics of cluster eating for diffusing clusters in a system of spatial dimension $d$ less than the upper critical dimension. By appealing to the analogy between cluster eating and the single-species reactions $A+A \rightarrow A$ and $A+A \rightarrow$ inert, for which the upper critical dimension is known to be 2 , we deduce that cluster eating 


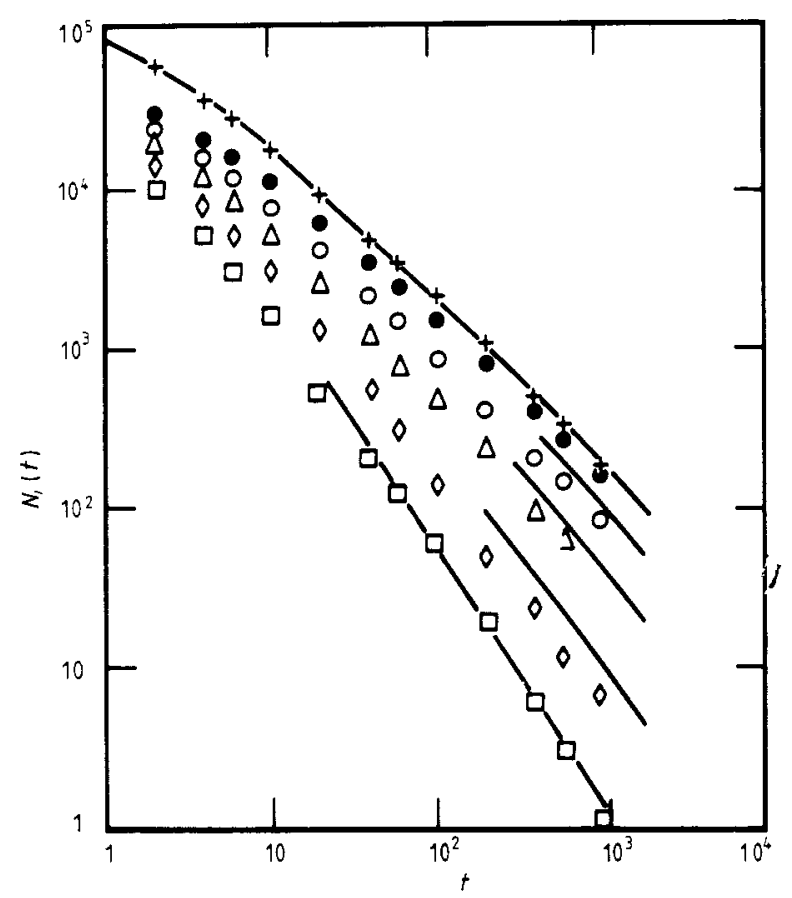

Figure 2. Typical simulation results in the mean-field limit. Shown is the case of a linear chain of 500000 sites in which the initial number of particles of mass $i, N_{1}(0)$, is 50000 for $1 \leqslant i \leqslant 5$. Plotted are $\Sigma_{\text {odd }} N_{1}(t)(+), N_{1}(t)(\bigcirc), N_{2}(t)(O), N_{3}(t)(\Delta), N_{4}(t)(\diamond)$ and $N_{5}(t)(\square)$. The full lines are the results from the asymptotic solution of (13).

also has an upper critical dimension equal to 2. Above two dimensions, fluctuations in cluster density can be ignored and the kinetics can be described accurately by the rate equation approach. Below two dimensions, however, fluctuations give rise to kinetic behaviour which is distinct from that of the mean-field limit. From the analogy with the single-species reactions, we now expect that the total density of clusters will decay as $t^{-d / 2}$.

To obtain more complete information, we turn to numerical simulations. Since the upper critical dimension is equal to 2 , we shall consider only the case of one dimension and typical results are shown in figure 3 . From the data we find that the total cluster density evidently decays as $t^{-1 / 2}$, as expected from the analogy with single-species reactions. Clusters with mass greater than 1 decay faster than $t^{-1 / 2}$, but we cannot find the asymptotic decay law from the numerical data alone. Work on theoretically predicting this decay is currently being done.

\section{Acknowledgments}

We are grateful to François Leyvraz for very helpful advice during the course of this work. We would also like to fondly remember Kiho Kang. This work was supported in part by a grant from the Army Research Office. DBA also acknowledges the support of the Weizmann Fellowship. 


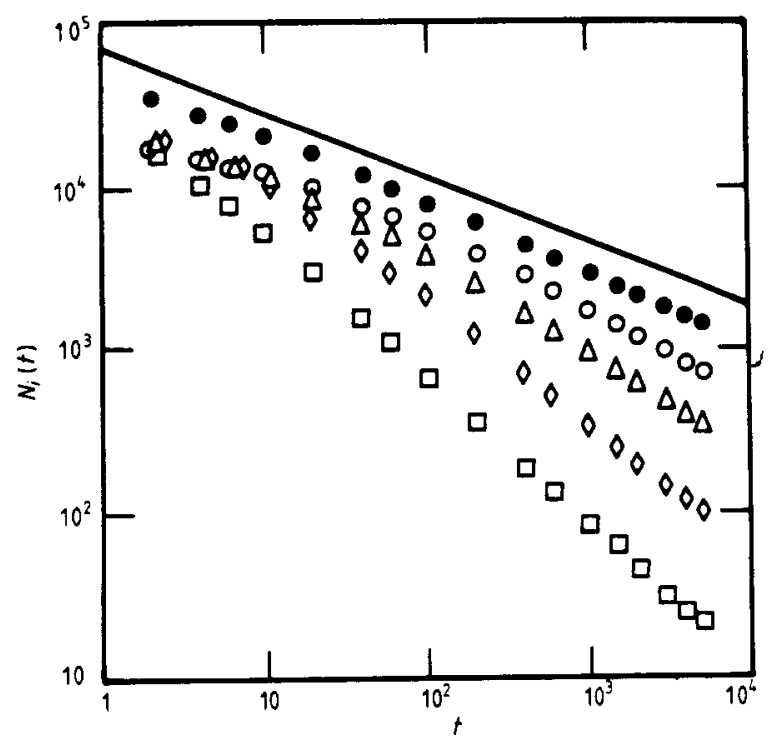

Figure 3. Simulation results for clusters undergoing nearest-neighbour hopping on a onedimensional chain of 500000 sites. Shown is the case of $N=5$. The initial number of particles of mass $i, N_{i}(0)$, is 50000 for $1 \leqslant i \leqslant 5$. Plotted are: $N_{1}(t)(O), N_{2}(t)(O), N_{3}(t)$ $(\triangle), N_{4}(t)(\diamond)$ and $N_{5}(t)(\square)$. The full line has slope $\frac{1}{2}$ and is shown for comparison.

\section{References}

Calef D F and Deutch J M 1983 Ann. Rev. Phys. Chem. 34493

Djordjevic Z and Meakin P 1986 J. Phys. A: Math. Gen. 19

Drake R L 1972 Topics in Current Aerosol Research ed G M Hidy and G R Brock (Oxford: Pergamon) Ernst M H 1985 Fundamental Problems in Statistical Physics VI ed E D G Cohen (Amsterdam: Elsevier) Kang K and Redner S 1985a Phys. Rev. A 32435 1985b Phys. Rev. A 302833

Kang K, Redner S, Meakin P and Leyvraz F 1986 Phys. Rev. A 331171

Leyvraz F 1984 Phys. Rev. A 29854

1985 J. Phys. A: Math. Gen. 18321

-1986 Proc. Cargese School 'On. Growth and Form' ed H E Stanley and N Ostrowsky (Boston, MA: Martinus Nijhoff) p 136

Leyvraz F and Redner S 1986 Phys. Rev. Lett. 57163

Lushnikov A 1973 J. Colloid Interface Sci. 48549

$1974 \mathrm{~J}$. Colloid Interface Sci. $\mathbf{4 8} 400$

1975 J. Colloid Interface Sci. 5494

Noyes R M 1961 Prog. Reac. Kinetics 1128

Torney D C and McConnell H M 1983a J. Phys. Chem. 871441

1983b Proc. R. Soc. A 387147

Toussaint D and Wilczek F $1983 \mathrm{~J}$. Chem. Phys. 782642

van Dongen P G D and Ernst M H 1985 Phys. Rev. Lett. 541396

Zumofen G, Blumen A and Klafter J 1985 J. Chem. Phys. 823198 